\title{
Analysis of crystal size and wavelength dependence using the deep UV laser processing crystals
}

\author{
Ayaka Harada ${ }^{1}$, Naohiro Matsugaki ${ }^{1,2}$, Toshiya Senda ${ }^{1,2}$
}

${ }^{1}$ Structure Biology Research Center, KEK, ${ }^{2}$ The Grad. Univ. for Advanced Studies, School of High Energy Accelerator Science

Native SAD phasing uses anomalous scattering signals from light atoms such as sulfur and phosphorus in protein crystals. The anomalous signals from these atoms are, however, much weaker than those from heavy atoms that are frequently utilized in protein crystallography. Therefore, high quality data collection is essential for native SAD phasing. Lower energy X-ray is favorable to enhance the anomalous signals but on the other hand X-ray absorption by sample, solvent, and air hinders the diffraction data collection. Here, we present a method minimizing the X-ray absorption; the solvent portion of a mounted frozen crystal is removed or the mounted crystal is spherically shaped by the deep UV laser processing technique (Kitano, 2004), (Murakami, 2004). According to an analysis of previous studies, data statistics were improved when the diffraction data for native SAD were collected with the deep UV laser processing crystals using the X-ray of $3.7 \mathrm{keV}$ at BL-1A of Photon Factory (Tsukuba, Japan).

In this study, crystals of BphA4 proteins which is ferredoxin reductase was used. BphA4 has an expected Bijvoet ratio $\left\langle\left|F_{\text {anom }}\right|\right\rangle /\langle|F|\rangle$ of $1.69 \%$, with 408 residues, including nine sulfur atoms. This experiment was carried out to compare crystal size and wavelength. We prepared $20 \mu \mathrm{m}$ and $70 \mu \mathrm{m}$ sized crystals from one BphA4 crystal and the diffraction data were collected using the X-ray of $3.7 \mathrm{keV}$ and $4.5 \mathrm{keV}$ at BL-1A (Figure). All diffraction data were collected with a helium beam path and a helium cold stream to reduce X-ray absorption by air. Each data was integrated using XDS (Kabsch, 2010). All data sets were merged using XSCALE (Kabsch, 2010). The merged data were input to autoSHARP (Vonrhein, 2007). In this time, we analyzed crystal size and wavelength dependence, so we report the result of that examination.

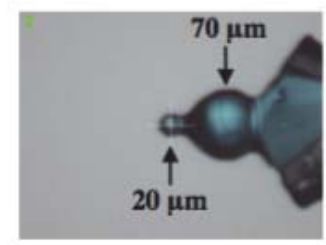

Figure. BphA4 processed crystal 